\title{
Might $\beta 3$-adrenergic receptor agonists be useful in disorders of glucose homeostasis?
}

\author{
Jeffrey S. Flier \\ Department of Medicine and Department of Neurobiology, Harvard Medical School, Boston, Massachusetts, USA.
}

\begin{abstract}
Brown and beige adipose tissues contain thermogenic fat cells that can be activated by $\beta 3$-adrenergic receptor agonists. In rodents, such drugs both diminish obesity and improve glucose homeostasis. In this issue of the $J C I$, O'Mara et al. and Finlin and Memetimin et al. report that chronic administration of the approved $\beta 3$ agonist mirabegron to human subjects was without effect on body weight or fat mass, but improved several measures of glucose homeostasis. Though the mechanisms mediating these metabolic effects are uncertain, the data suggest that $\beta 3$ agonists could have therapeutic utility in disorders of glucose homeostasis.
\end{abstract}

\section{Activated brown adipose tissue} When rodents are exposed to environmental cold, their brown adipose tissue (BAT) becomes activated, generating heat required for thermal homeostasis (1). The physiologic loop involves cold-induced activation of the sympathetic nervous system, which then releases noradrenaline from nerve terminals to activate $\beta 3$-adrenergic receptors ( $\beta 3$-ARs) on BAT cell membranes. Activating uncoupling protein 1 (UCP1) on the inner mitochondrial membrane then uncouples mitochondrial respiration to oxidize substrate and generate heat. A subpopulation of white adipose tissue (WAT) cells called "brite" or "beige" cells have a distinct lineage and gene expression profile and are also capable of displaying induced, uncoupled respiration through UCP1 (2). The relative contributions of BAT versus beige adipose tissues to physiology is poorly defined, and additional heterogeneity of brown adipocyte cells has recently emerged (3).

Evidence that altered BAT function contributes to metabolic disorders has derived from loss- and gain-of-function approaches. When chronically exposed to high-calorie (obesogenic) diets, mouse
BAT becomes hypertrophied and activated. This was proposed to limit diet-induced obesity, suggesting a role for BAT in physiologic resistance to obesity via diet-induced thermogenesis (4). Supporting this hypothesis, a transgene-induced reduction in BAT mass in mice produced obesity, and these mice had further increased susceptibility to obesity on obesogenic diets $(5,6)$. Pharmacologic activation of $\beta 3$-ARs on rodent BAT cells stimulated this tissue, increasing uncoupled respiration and energy expenditure, while reducing obesity and improving glucose homeostasis (7). Taken together, these results suggested the potential utility for this class of drugs in obesity and type 2 diabetes.

Though quite well established in rodents, the role of BAT/beige cells in human physiology is less well understood. Human infants have BAT, as do adults chronically exposed to cold stress, patients with pheochromocytoma and consequent adrenergic excess, and some healthy humans at thermoneutrality $(1,8)$. But the physiologic role of BAT and its capacity to be activated for therapeutic benefit remain uncertain. This is not for lack of effort.
Many companies have sought to develop $\beta 3$ agonists for metabolic indications, but these failed for a variety of reasons, including lack of $\beta 3$ specificity causing cardiovascular toxicity, poor bioavailability, and/or insufficient BAT/beige tissue to mediate therapeutic effects (9). Consequently, the development of $\beta 3$ agonists for metabolic indications has largely been abandoned.

One stimulus to reinvestigating this pathway is the availability of mirabegron, a $\beta 3$ agonist approved for human use in the treatment of overactive bladder. Initially developed for metabolic indications, the drug was repurposed when metabolic studies stalled and $\beta 3$ receptors were identified in bladder smooth muscle, where their engagement suppressed overactive muscle (10).

\section{Improving glucose and insulin homeostasis}

Two articles in this issue of the JCI provide evidence that chronic treatment of human subjects with mirabegron improves glucose and insulin homeostasis $(11,12)$. Though both studies are preliminary and neither is placebo controlled, they could rekindle 40-year-old hopes for utility of this pathway in metabolic disease (Figure 1).

The study by O'Mara et al. is straightforward (11). Fourteen young, healthy female subjects received $100 \mathrm{mg}$ mirabegron daily for 4 weeks, which is twice the approved dose. The primary endpoint was BAT metabolic activity assessed by combined PET/CT with $\left[{ }^{18} \mathrm{~F}\right]$-2-fluoro-D2-deoxy-D-glucose ( ${ }^{18} \mathrm{~F}$-FDG), and this validated functional measure increased 2-fold, along with a $5.8 \%$ increase in resting energy expenditure. So far, so good. If the goal of negative energy balance had 


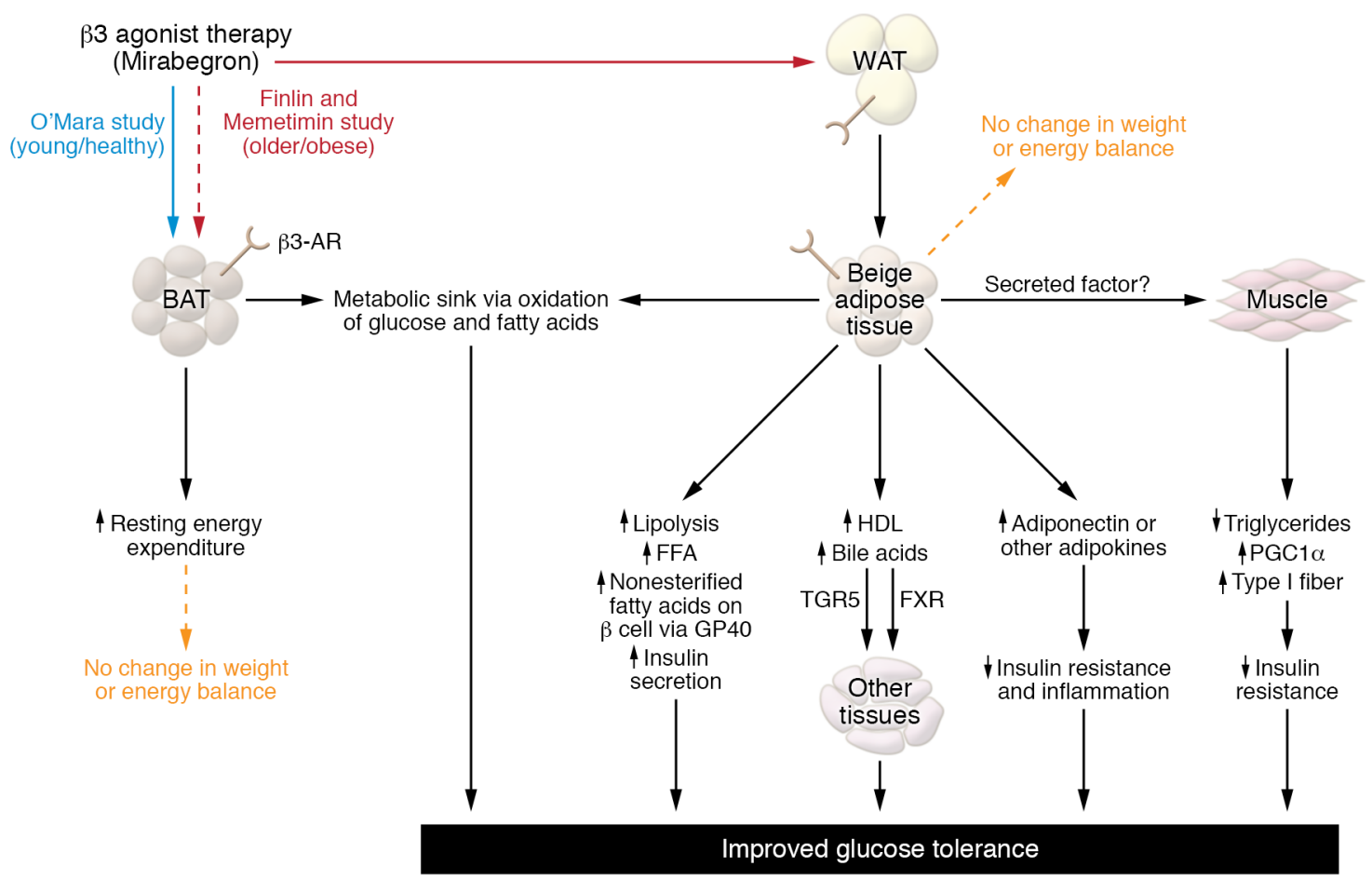

Figure 1. Model for $\beta$ 3-AR agonist therapeutic utility in glucose homeostasis. Mirabegron administered to young, healthy subjects or older subjects with obesity may stimulate $\beta 3$-ARs on BAT or WAT. FFA, free fatty acid.

been achieved, reduced body weight and fat mass would have been expected, but, perhaps surprisingly, neither changed, suggesting some form of compensation. However, all was not lost, as treatment did improve insulin sensitivity, glucose effectiveness, and insulin secretion in these healthy subjects as determined by intravenous glucose tolerance tests. Levels of the fat-derived antidiabetic and antiinflammatory adipokine adiponectin increased, providing one potential explanation for these metabolic effects.

Finlin and Memetimin et al. studied thirteen older, obese, insulin-resistant subjects receiving the highest approved dose of mirabegron (50 mg) for 12 weeks (12). In addition to the outcomes measured by O'Mara et al., the Mimetimin et al. study included biopsies of WAT and skeletal muscle. And like O'Mara and researchers, they observed improved glucose and insulin homeostasis but no treatment-induced changes in body weight or composition. Unlike in the O'Mara study, subjects in the Finlin and Memetimin study showed no induction of BAT activity, as assessed by PET/CT, and no change in energy expenditure or adiponectin levels. The results of tissue biopsies were interesting. WAT had molecular markers of browning and changes in gene expression consistent with improved adipose cell function. Although skeletal muscle lacks $\beta 3$ receptors, this tissue showed reduced triglycerides, increased expression of PPAR $\gamma$ coactivator $1 \alpha$ (PGC1 $\alpha)$, and increased appearance of type I fibers. To explore a potential mechanism, conditioned media from mirabegron-treated adipocytes were added to cultured human muscle cells. In this system, PGC1A expression was induced, suggesting that an unidentified factor secreted by $\beta 3$-stimulated BAT/ beige cells could have brought about these effects in muscle cells (12).

\section{Clinical implications}

What do these studies suggest regarding the physiology of BAT and beige cells and the therapeutic potential for $\beta 3-A R$ agonists in human obesity and diabetes? Dosing young, healthy females with this $\beta 3$-AR agonist (at a dose greater than the approved one that causes a cardiovascular signal) produced no change in energy balance despite increased BAT activity and total energy expenditure (11), consis- tent with evidence that functional BAT can be induced by environmental cold exposure, but casting doubt on the utility of this approach for achieving weight loss (13). Subjects with obesity and disordered glucose homeostasis showed browning of WAT and improved metabolic parameters with mirabegron, without altered body weight or composition, BAT function by PET/CT, or energy expenditure (12). This is consistent with evidence that age and obesity are associated with reduced BAT function $(14,15)$ and further suggests that the improved glucose homeostasis in these subjects may be a consequence of activating beige adipocytes rather than BAT.

These actions raise additional mechanistic questions. Which tissues expressing $\beta 3$ receptors mediate these metabolic actions? In prior research, activating BAT with cold exposure increased glucose disposal in proportion to BAT activation, with BAT perhaps serving as a metabolic sink via increased oxidation of fatty acids and glucose (13). Since in the study by Finlin and Memetimin et al. $\beta 3$ activation produced metabolic effects without BAT activation, white or beige adipocytes rather than BAT may have been the key $\beta 3$ target 
tissue in these subjects. Apart from acting as a metabolic sink via substrate oxidation, how else might $\beta 3$-activated brown or beige adipocytes affect tissues lacking $\beta 3$-ARs, such as muscle, $\beta$ cells, or other tissues? Several potential mechanisms can be considered (Figure 1). $\beta 3$ agonists may stimulate the release of metabolically active adipokines such as adiponectin. Insulin secretion might result from the lipolysis-induced rise in nonesterified fatty acids acting via GRP40, a fatty acid receptor on $\beta$ cells (16). Since $\beta 3$ agonist treatment increased HDL and circulating bile acids, the latter could then act through the farnesoid $\mathrm{X}$ receptor (FXR) and/or the G protein-coupled bile acid receptor TGR5 to benefit metabolism (17). Finally, a beige fat-derived factor that induces PGC1A and fiber switching in skeletal muscle could improve metabolism (12).

Taken together, these studies suggest that chronic treatment with a $\beta 3$ agonist, despite uncoupling brown or beige fat respiration and even inducing energy expenditure in healthy subjects, has limited or no ability to induce negative energy balance and weight loss in humans, as these agents clearly do in mice. Whether this is the result of compensatory increases in energy intake or some other mechanism is unknown. But despite the disappointment of failing as an antiobesity therapeutic, these studies could renew interest in the use of $\beta 3$ agonists to treat impaired glucose homeostasis. Furthering that goal will require larger, placebo-controlled trials and deeper insight into mechanisms by which activation of $\beta 3$ receptors on beige adipocytes or other cells produce metabolic benefits, especially in metabolically important tissues lacking the receptors, such as muscle, liver, and $\beta$ cells. So, at this moment, the potential therapeutic utility of $\beta 3$ agonists for metabolic disorders is down, but it's not yet out.

Address correspondence to: Jeffrey S. Flier, 220 Longwood Ave., Goldenson 542, Boston, Massachusetts 02115, USA. Phone: 617.432.1501; Email: Jeffrey_flier@ hms.harvard.edu.

1. Cannon B, Nedergaard J. Brown adipose tissue: function and physiological significance. Physiol Rev. 2004;84(1):277-359.

2. Kajimura S, Spiegelman BM, Seale P. Brown and beige fat: physiological roles beyond heat generation. Cell Metab. 2015;22(4):546-559.

3. Song A, et al. Low- and high-thermogenic brown adipocyte subpopulations coexist in murine adipose tissue. J Clin Invest. 2020;130(1):247-257.

4. Rothwell NJ, Stock MJ. A role for brown adipose tissue in diet-induced thermogenesis. Nature. 1979;281(5726):31-35.

5. Lowell BB, et al. Development of obesity in transgenic mice after genetic ablation of brown adipose tissue. Nature. 1993;366(6457):740-742.

6. Hamann A, Flier JS, Lowell BB. Decreased brown fat markedly enhances susceptibility to diet-induced obesity, diabetes, and hyperlipid- emia. Endocrinology. 1996;137(1):21-29.

7. Liu X, Pérusse F, Bukowiecki LJ. Mechanisms of the antidiabetic effects of the beta 3-adrenergic agonist CL-316243 in obese Zucker-ZDF rats. Am J Physiol. 1998;274(5):R1212-R1219.

8. Cypess AM, et al. Identification and importance of brown adipose tissue in adult humans. $N$ Engl JMed. 2009;360(15):1509-1517.

9. Arch JR. Challenges in $\beta(3)$-adrenoceptor agonist drug development. Ther Adv Endocrinol Metab. 2011;2(2):59-64.

10. Edmondson SD, et al. Discovery of vibegron: a potent and selective $\beta 3$ adrenergic receptor agonist for the treatment of overactive bladder. JMed Chem. 2016;59(2):609-623.

11. O'Mara AE, et al. Chronic mirabegron treatment increases human brown fat, HDL cholesterol, and insulin sensitivity. J Clin Invest. 2020;130(5):2209-2219.

12. Finlin BS, et al. The $\beta 3$-adrenergic receptor agonist mirabegron improves glucose homeostasis in obese humans. JClin Invest. 2020;130(5):2319-2331.

13. Chondronikola $\mathrm{M}$, et al. Brown adipose tissue improves whole-body glucose homeostasis and insulin sensitivity in humans. Diabetes. 2014;63(12):4089-4099.

14. Saito M, et al. High incidence of metabolically active brown adipose tissue in healthy adult humans: effects of cold exposure and adiposity. Diabetes. 2009;58(7):1526-1531.

15. Vijgen GH, Bouvy ND, Teule GJ, Brans B, Schrauwen P, van Marken Lichtenbelt WD. Brown adipose tissue in morbidly obese subjects. PLoS ONE. 2011;6(2):e17247.

16. Pang Z, et al. GPR40 is partially required for insulin secretion following activation of beta3-adrenergic receptors. Mol Cell Endocrinol. 2010;325(1-2):18-25.

17. Broeders EP, et al. The bile acid chenodeoxycholic acid increases human brown adipose tissue activity. Cell Metab. 2015;22(3):418-426. 